\section{The Heterodyne Null Method of Measuring Dielectric Gonstant.}

Ir is found that different investigators using the same heterodyne null method differ widely in their results for the same substance. This is indicated from the results of different investigators in the cases of carbon dioxide, hydrogen sulphide, and methyl chloride. In a recent paper (Phys. Rev., $32 ; 1928$ ) Zahn has tried to find out the sources of this discrepancy, and suggests that besides the calibration errors there are other possible sources of error in the method. From the data of Watson (Proc. Roy. Soc., 117; 1927), Zahn attributes one of these to the difference in frequency of the oscillating circuits which the various investigators have used. But this cannot produce a large effect on the results. We wish to point out the possible sources of error from our own experience for the last couple of years with the apparatus we are using for measurements of dielectric constant. They are as follows :

(1) The method of measuring temperature by winding the platinum wire on the glass tube containing the experimental condenser or on the condenser itself (when a cylindrical condenser is used) with proper insulation, may probably produce a large error owing to the presence of this stray capacity near the experimental condenser if proper care is not taken to eliminate this effect. Even if the bath which contains the experimental condenser is well earthed, still this stray capacity affects the results, and proper care should be taken to connect the experimental condenser in the case of a cylindrical one. This effect has been carefully studied by Sarkar (Ind. Jour. Phys., 3 ; 1928). He found that when the inner cylinder of the experimental condenser was connected to the anode of the thermionic tube it gave more concordant results than when the outer cylinder was connected. In the latter case even the movement of any other conductor or variation of current in any other circuit connected to the mains in the room would change the pitch of the beat note, whereas no such difficulty was observed in the former case.

Hence the method of measuring the temperature by the thermo-couple is preferable. It is also found that when the thermo-couple is put into the bath the frequency of the oscillating circuit changes. It is therefore advisable not to put the thermo-couple into the bath at the time of taking observations, and the temperature should be measured just before and after the observations are taken.

(2) If the bath be heated electrically from the mains, it is found that there is a difference of pitch when there is no current passing through the heating coil and when there is a current passing. This evidently indicates a presence of stray capacity and induction effect. To avoid this, it is better to disconnect the heating coil of the bath from the main when the measurement is to be taken.

Taking all these precautions we have found that the value of the dielectric constant for dry and carbon dioxide free air at N.T.P., is $1 \cdot 000579_{ \pm 4}$.

$$
\text { P. N. Ghosh. }
$$$$
\text { P. C. Mahanti. }
$$

University College of Science and Technology, Calcutta.

\section{The Swelling of Rubber.}

Some recent experiments on the diminution of the vapour pressure of solvents in rubber jellies as well as measurements of swelling pressure allow us to draw some conclusions as to the nature of the swelling phenomenon.

It has been found that, at the same concentration of rubber, the vapour pressure of rubber jellies from rubber of different origin was the same, and previous mechanical working (mastication) of rubber had also no effect on this value (P. Stamberger, Rec. Trav. Chim. Pays-Bas., 47, 316; 1928). After the mechanical working, however, the rubber swells in an unlimited manner and gives as a resulting product up to a concentration of 30 per cent a more or less viscous liquid. This behaviour shows that the solvent is not bound by surface adsorption and that there is a great resemblance to the process of molecular disperse solution. The three solvents used for these determinations were: carbon disulphide, chloroform, and benzene. The same relative vapour pressure diminution was found when the concentration has been calculated as grams of rubber in $1 \mathrm{gm}$. mol. solvent.

The shape of the curve in which the relative vapour pressure was plotted against the concentration differs from that expected according to Raoult's laws. The curves bend rapidly at a concentration of $20-30$ per cent towards the vapour pressure axis. Although some resemblance to the ordinary process of solution was found, this behaviour suggests a process of a more complicated nature. But the affinity of the solvent for the rubber can only be due to molecular forces.

Although no difference has been found between the vapour pressure of the 'liquid' jelly of masticated rubber and the solid elastic jelly of untreated rubber, the swelling pressure of both showed big differences.

The liquid, masticated rubber jelly had a swelling pressure of $35 \mathrm{~cm}$. of mercury at a concentration of $0.4 \mathrm{gm}$. rubber in 1 c.c. of solvent (toluene), the unmasticated had the same swelling pressure at a much lower concentration, $0 \cdot 11 \mathrm{gm}$. $/ \mathrm{c} . \mathrm{c}$.

This value shows that the swelling of a structureless (masticated) rubber differs greatly from the swelling of a rubber having a definite structure, although both show the same vapour pressure diminution. For this reason it is not possible to calculate the swelling pressure from the vapour pressure diminution on a simple thermodynamic basis.

This behaviour shows that the forces which cause the imbibition of solvent by the jelly are not of a uniform nature and the 'structure factor' has an influence when the solvent is present in a liquid form (P. Stamberger, loc. cit.).

Particulars of this investigation will be published shortly.

P. Stamberger.

University College, London, C. M. BLow. June 4.

\section{The Isotopes of Oxygen.}

I HAVE recently been studying the vibrational energy functions of molecules, in connexion with a recalculation of heats of dissociation, and have noticed that the equation given by Dieke and Babcock (Proc. Nat. Acad. Sci., 13, 670; 1927) for the upper level of the atmospheric bands of oxygen has an unusually large coefficient for the $n^{3}$ term. This, I now find, is due to an arithmetical error of $2 \mathrm{~cm} .^{-1}$ in their location of the origin of the $O-O$ band. The correct figure should be $13,120.97 \mathrm{~cm}^{-1}$, instead of their 13,122.97. The correction not only leads to the expected small coefficient for $n^{3}$, but also brings their data into good agreement with the older constants, as given on p. 232 of the " Report on Molecular Spectra in Gases". The resulting corrected equation is

$$
E_{n}=13,120 \cdot 97+1418 \cdot 69 n-13 \cdot 925 n^{2}-0 \cdot 02 n^{3}
$$

$$
(n=0,1,2,3) \text {, }
$$

as contrasted with their

$$
E_{n}=13,122 \cdot 965+1415 \cdot 017 n-11 \cdot 911 n^{2}-0 \cdot 3525 n^{3}
$$

The new constants are of importance in connexion with the isotopes of oxygen. Giauque and Johnston

$$
\text { No. 3114, Vou. 124] }
$$

